\title{
14
}

\section{Selective Generation of Symmetrical Test Cases}

\author{
F. Michel and P. Azéma and K. Drira \\ $L A A S-C N R S$ \\ 7, av. du Colonel Roche, 31077 Toulouse Cedex, France. \\ Phone: (+33) 61-33-62-00. Fax: (+33) 61-33-62-65. \\ email: $\{$ fmichel, azema, drira\}@laas.fr
}

\begin{abstract}
In this paper, system symmetries are shown to efficiently reduce the size of the complete test suite required for checking the implementation conformance. The reduction is obtained by eliminating all the symmetrical test cases during the generation of the test suite. The method is applied over the theoretical model of conformance testing developed by [Bri88] using the implementation relation conf. In this model, symmetries are defined as bijective renamings of actions which preserve conformance properties, and allow test folding. The symmetries may concern either architectural properties, or execution sequences, or data structures, respectively. By exploiting data or state symmetries, infinite complete test suites may be reduced into finite one.
\end{abstract}

\section{Keywords}

Generation of test cases, conformance testing, symmetrical systems

\section{INTRODUCTION}

This study merges two topics : conformance testing and validation of symmetrical systems.

Conformance testing concerns the process of checking whether a system implementation conforms to its specification when this implementation is only accessed by its interaction points (black box hypothesis).

On the other hand, the validation of symmetrical system [PHJ91] has been studied in the field of verification where behaviour descriptions are freely available. Several dedicated formalisms [Lam91, ID93] have been designed to verify structural properties [Lam91, Sch94] or temporal logic formulae by means of model checking [ECJ93, EE93, EE95].

The main motivation of these works lies in the reduction, modulo system symmetries, 
of the state space made untractable by combinational explosion. In testing framework, this motivation can be reformulated as : how to reduce the size of the test suite required for testing the implementation conformance.

The reduction process is rather simple. Consider, for instance, the transport protocol between two entities $E_{1}$ and $E_{2}$. If the implementation of both entities are exactly the same, then testing the connection establishment by $E_{2}$ is worthless if the connection establishment by $E_{1}$ has already been tested : an architectural symmetry exists between $E_{1}$ and $E_{2}$. In the same way, if the implementation of a buffer behaves independently of conveyed values then one needs only to test the buffer for one particular value : a data symmetry exists among all the values. More generally, if one knows system symmetries, then one can eliminate all test cases symmetrical to an already generated test case.

Though this approach is closed to test selection [BTV91] regarding the expected goal, it differs from the latter in three points : first, it is performed during the generation of the test suite, then, it does not use external knowledges but extra informations : system symmetries, finally, it does not weaken the conformance relation since only redundancies caused by symmetries will be eliminated. That is why, our approach could be identified as a selective generation of test cases by means of system symmetries.

Formally, the chosen theoretical framework is the model of synchronous testing developed by [Bri88, Tre89] over LOTOS. In fact, we will use the version of [Tre92] where systems are described by labelled transition systems (LTS). The implementation relation to be tested is the relation conf. conf is defined from testable properties $S$ after $\sigma$ must A. $S$ after $\sigma$ must $\mathbf{A}$ means that every states reached after the sequence of actions $\sigma$ in the system $\mathbf{S}$ must accept an action in the set $\mathbf{A}$.

A complete test suite for conf can be generated automatically as an unique LTS, the so-called canonical tester. This derivation is based on the construction of an intermediate object : the failure tree or its complementary version the success tree (remark: this is not exactly the acceptance tree defined in [Hen85]).

Finally, the canonical tester is applied in a synchronous way to the implementation and a verdict is returned by observing deadlocks over the composition.

This paper defines a symmetrically selective generation of test cases within this model.

First, in order to study what are the possible consequences of a selective generation, we will parameterize the whole method by sets of properties to be tested; these sets will be called domains. Therefore, the implementation relation will be denoted $\leq_{D}$ where $\mathrm{D}$ is an arbitrary domain of testable properties, ( conf corresponds to a particular domain ). $I \leq D S$ means that if $\mathrm{S}$ verifies a property in $\mathrm{D}$ then I verifies the same property. The main result is that if $D$ is a domain of properties verified by the specification and if $D$ can be represented by a success tree then the whole generation method is still valid. Besides, we notice that if $D^{\prime} \subseteq D$ then the test suite required for $\leq_{D^{\prime}}$ will be smaller than the test suite required for $\leq_{D}$. So, in this context, a selective generation of test cases consists in finding a subset D' of the initial domain D such that :

- $I \leq_{D} S \Leftrightarrow I \leq_{D^{\prime}} S$ (the reduction does not weaken the implementation relation)

- D' can be represented by a success tree if $D$ can be represented by a success tree (if the generation of test cases can be applied over D then it can be applied over D')

The reduction will be achieved by means of symmetries. 
Symmetries are defined over a LTS by means of bijective renamings of actions : two states in the system are symmetrical iff they verify the same testable properties up to a particular renaming of actions, two sequences of actions are symmetrical iff they reach symmetrical states. Then the notion of group of symmetries allow us to build the equivalence relation "is equal up to a symmetry".

In the case of conformance testing, two LTS are studied : the implementation and the specification. That is why, the hypothesis (H1) is added:" (H1) : the considered symmetries are common to the specification and the implementation".

The reduced domain of properties, D', is defined as a set of representatives of D for the equivalence relation "is equal up to a common symmetry". D' fulfills both requirements : under hypothesis (H1), $I \leq_{D} S \Leftrightarrow I \leq_{D^{\prime}} S$ and a quotient success tree representing D' can be build from a success tree attached to D by cutting symmetrical branches. Consequently, the canonical tester derived from the quotient success tree represents a reduced complete test suite for $\leq_{D}$.

We may note that our method relies strongly on (H1), but how to identify symmetries over a partially-known implementation ? In fact, (H1) can be established by exploiting three kind of informations about the system : architectural, data and state symmetries.

Data and state symmetries are particularly interesting since they allow us to test systems lying beyond the operational scope of the initial method. Indeed, a finite-branching success tree reduced by data symmetries may be computed even if the initial success tree is infinite-branching. In the same way, a finite-depth success tree reduced by state symmetries may be processed even if the initial success tree has an infinite depth.

In this paper, symmetry handling is assumed to be supported by the designer. That is, the designer is able to provide group of symmetries and to implement a decision procedure for the relation "is equal up to a symmetry".

The remaining of the document is organized as follows: section 2 recalls basic notions about LTS, section 3 explains the parameterized test model, section 4 introduces symmetries into this model, section 5 details the symmetrically selective generation, section 6 instantiates our results in the case of architectural, data and state symmetries.

\section{LABELLED TRANSITION SYSTEMS}

Let us consider $\mathrm{L}$ a set of (visible) actions and $\tau$ a particular symbol representing the internal computing. $L^{*}$ denotes the set of action sequences, $\epsilon$ the empty sequence. $\mathcal{P}(L)$ denotes the power set of L. $|\sigma|$ denotes the length of a sequence $\sigma$.

A labelled transition system (LTS), B, is a triple $\left(\mathcal{Q}, \rightarrow, s_{0}\right)$ where $\mathcal{Q}$ is the set of states, $s_{0}$ is the initial state, and $\rightarrow \subseteq \mathcal{Q} \times L \cup\{\tau\} \times \mathcal{Q}$ is the transition relation. $\mathcal{Q}$ and $\mathrm{L}$ need not be finite.

We note $: \forall a \in L: s \stackrel{a}{\Longrightarrow} s^{\prime} \stackrel{\text { def }}{\Leftrightarrow} s \stackrel{\tau^{*} a \tau^{*}}{\longrightarrow} s^{\prime}$ (abstraction of the internal computing)

$$
\begin{aligned}
& \forall \sigma=a_{1} \ldots \ldots a_{n} \in L^{*}: s \stackrel{\sigma}{\Longrightarrow} s^{\prime} \stackrel{\text { def }}{\Leftrightarrow} s \stackrel{a_{1}}{\Longrightarrow} \ldots \stackrel{a_{n}}{\Longrightarrow} s^{\prime} \quad \text { and } \quad s \stackrel{\epsilon}{\Longrightarrow} s^{\prime} \stackrel{\text { def }}{\Leftrightarrow} s=s^{\prime} \text { or } s \stackrel{\tau^{*}}{\longrightarrow} s^{\prime} \\
& s \stackrel{\sigma}{\Longrightarrow} \stackrel{\text { def }}{\Leftrightarrow} \exists s^{\prime}: s \stackrel{\sigma}{\Longrightarrow} s^{\prime} \quad B \stackrel{\sigma}{\Longrightarrow} s \stackrel{\text { def }}{\Leftrightarrow} s_{0} \stackrel{\sigma}{\Longrightarrow} s \\
& B \stackrel{\sigma}{\Longrightarrow} \stackrel{\text { def }}{\Leftrightarrow} s_{0} \stackrel{\sigma}{\Longrightarrow} \quad \text { Trace }(B)==_{\text {def }}\left\{\sigma \in L^{*} / B \stackrel{\sigma}{\Longrightarrow}\right\} \quad \text { out } t_{B}(s)=_{\text {def }}\{a / s \stackrel{a}{\Longrightarrow}\} \text {. }
\end{aligned}
$$

A deterministic tree $\mathrm{B}$ is a particular LTS such that the relation $\{(s, \sigma) / B \stackrel{\sigma}{\Longrightarrow} s\}$ 
is one-to-one. In this case, a state is called a node, $|\sigma|$ is the depth of $\mathrm{s}$, and the set of states whose depth is $\mathrm{k}$, is the $k^{\text {th }}$ level of the tree.

Remark : transition relations $\rightarrow$ and $\Rightarrow$ are equal over a deterministic tree.

In the sequel, S (resp. I) is the specification (resp. implementation) LTS.
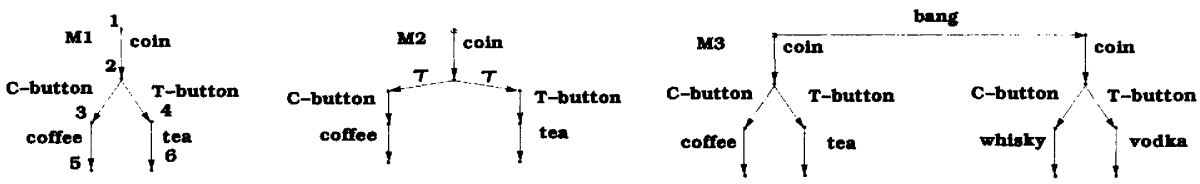

Figure 1 LTS of vending machines

Example : LTS M1, M2, M3 in figure 1 describes three vending machines. States in M1 are numbered. The deterministic tree, $\mathbf{M} 1$, is a vending machine delivering either coffee or tea for one coin. The selection is performed by pushing the right button. The machine M2 decides internally whether you can have coffee or tea. M3 adds a new behaviour to M1 : when you kick the machine (bang), it starts delivering whisky or vodka instead of respectively coffee or tea.

\section{SYNCHRONOUS TESTING}

Conformance testing consists in determining whether the implementation conforms to its specification by only using implementation interaction points with the environment. In synchronous testing, test cases are run in a synchronous way over the implementation. Here, the interactions are represented by the visible actions of the LTS.

In this section, implementation relations together with test generation defined in [Tre92] will be parameterized.

\subsection{Parameterized implementation relations}

The testable properties are after $\sigma$ must $\mathbf{A}$ where $\sigma \in L^{*}$ and $\mathbf{A} \in \mathcal{P}(L)$. A LTS, B, satisfies after $\sigma$ must $\mathbf{A}$, we note $B$ after $\sigma$ must $\mathbf{A}$, iff :

$$
\forall s: B \stackrel{\sigma}{\Longrightarrow} s \Rightarrow \exists a \in \mathbf{A}: s \stackrel{a}{\Longrightarrow}
$$

Intuitively, $B$ after $\sigma$ must $\mathbf{A}$ means that B must accept an action in $\mathbf{A}$ after $\sigma$.

Example : The following properties are satisfied by the vending machines :

M1 after coin must \{T-button\} M3 after coin must \{bang,C-button\}

But M2 does not satisfy after coin must $\{\mathrm{T}$-button\}.

The relations specifying the implementation conformance are called implementation relations. They are all built according to the same pattern. First, choose a domain of pairs $(\sigma, \mathbf{A})$ (the properties to be tested) then claim that I conforms to $\mathrm{S}$ iff all properties in $\mathrm{D}$ satisfied by $\mathrm{S}$ are also satisfied by I. Formally :

Definition 31 (Implementation Relations) Let $D \subseteq L^{*} \times \mathcal{P}(L)$ be a domain $I \leq_{D} S \stackrel{\text { def }}{\Leftrightarrow} \forall(\sigma, \mathbf{A}) \in D: S$ after $\sigma$ must $\mathbf{A} \Rightarrow I$ after $\sigma$ must $\mathbf{A}$

Remark: the conf relation is expressed by : $I$ conf $S \stackrel{\text { def }}{\Leftrightarrow} I \leq_{\operatorname{race}(S) \times \mathcal{P}(L)} S$ 
Our aim is to reduce D to the smaller domain D' such that $I \leq_{D} S \Leftrightarrow I \leq_{D^{\prime}} S$. Symmetries will be exploited for this purpose, nevertheless structural reductions are already possible. Let $\mathrm{D}$ and $\mathrm{D}$ ' be two domains, we note :

$S[D]=_{\text {def }}\{(\sigma, \mathbf{A}) /(\sigma, \mathbf{A}) \in D$ and $S$ after $\sigma$ must $\mathbf{A}\}$ (prop. in D satisfied by $\mathrm{S}$ )

$D^{\prime} \sqsubseteq D \stackrel{\text { def }}{\Leftrightarrow}\left(D^{\prime} \subseteq D\right.$ and $\left.\forall(\sigma, \mathbf{A}) \in D, \exists\left(\sigma, \mathbf{A}^{\prime}\right) \in D^{\prime}: \mathbf{A}^{\prime} \subseteq \mathbf{A}\right)$

Then, it holds that:

\section{Property 31 (Structural Reductions)}
(1) $I \leq_{D} S \Leftrightarrow I \leq_{S[D]} S \quad$ and
(2) $\forall D^{\prime} \sqsubseteq S[D]: I \leq_{D} S \Leftrightarrow I \leq_{D^{\prime}} S$

Sketch of proof : (1) As False $\Rightarrow$ Any, only properties satisfied by $S$ are discriminating.

(2) From : $\forall L T S B: \mathbf{A}^{\prime} \subseteq \mathbf{A}$ and $B$ after $\sigma$ must $\mathbf{A}^{\prime} \Rightarrow B$ after $\sigma$ must $\mathbf{A}$

The test generation takes benefit of these reductions.

\subsection{Parameterized test generation}

The derivation takes as input a particular tree, the success tree. The success tree is a compressed form of the domain D. Part 1 deals with the notion of success tree. Part 2 recalls briefly the process of synchronous testing (derivation, application, verdict). Part 3 presents the fundamental result about the validity of the parameterized test generation. Part 4 shows how the parameterized test generation is instantiated when $\leq_{D}$ is conf.

\section{Success trees}

A success tree $\mathrm{F}$ is a deterministic tree labelled by a mapping must $_{F}()$ such that :

$$
\forall s \text { node : } \operatorname{must}_{F}(s) \subseteq \mathcal{P}(L) \quad \text { and } \operatorname{out}_{F}(s) \subseteq \cup_{\mathbf{A} \in \text { must }_{F}(s)} \mathbf{A}
$$

For instance, figure 2 depicts three success trees.

Success trees are compressed forms of domains. Indeed, a domain D can be attached to each success tree $\mathrm{F}$ in this way:

$$
(\sigma, \mathbf{A}) \in D \Leftrightarrow\left(\exists s: F \stackrel{\sigma}{\Longrightarrow} s \text { et } \mathbf{A} \in \operatorname{must}_{F}(s)\right)
$$

On the other hand, some domains may not be represented by a success tree. For instance $\{(a,\{b\}),(a c,\{b\})\}$ cannot be associated with any success tree, since the condition out $_{F}(s) \subseteq \cup_{\mathbf{A} \in \text { must }_{F}(s)} \mathbf{A}$ will never be fulfilled.

\section{Test Derivation and application}

As this process will not be modified by the symmetrical method, we will only recall its principles. However, a more detailed presentation can be found in appendix 1 .

It is assumed that a success tree, $\mathrm{F}$, attached to the domain $\mathrm{D}$ is available. First, a complete test suite can be systematically derived from $\mathrm{F}$. This test suite is computed as a particular LTS called the canonical tester. Then, the canonical tester is synchronized with the implementation over their common actions. Finally, a verdict is returned by observing the resulting deadlocks. The verdict is a success iff all the observed deadlocks are caused by the canonical tester.

In the sequel, the whole process is denoted by a function Test(F,I). The function outputs "success" iff the obtained verdict is a success. 


\section{Fundamental result}

The validity of the method relies on the following theorem :

Theorem 31 If $D$ is a domain of properties satisfied by $S$ and if $F$ is a success tree representing $D$ then : $\mathbf{T e s t}(\mathbf{F}, \mathbf{I})$ outputs "success" iff $I \leq_{D} S$

The proof relies on the same arguments that in [Tre92].

\section{Testing conf}

For conf, a success tree can be computed automatically from the specification $\mathrm{S}$.

At first, the underlying deterministic tree associated with the sequences in Trace $(S)$ is built : for all $\sigma$ in Trace( $\mathrm{S}$ ), a node, $\hat{\sigma}$, is created in $\mathrm{F}$, and the transition relation in $\mathrm{F}$ is defined by : $\forall \sigma, \sigma . a \in \operatorname{Trace}(S): \hat{\sigma} \stackrel{a}{\longrightarrow} \widehat{\sigma . a}$.

Then, the mapping must $_{F}$ is processed by : must $_{F}(\hat{\sigma})=_{\text {def }} \Psi\left(O U T_{S}(\sigma)\right)$ where $\operatorname{OUT}_{S}(\sigma)=_{\text {def }}\left\{\right.$ out $\left._{S}(s) / S \stackrel{\sigma}{\Longrightarrow} s\right\}$, and $\Psi$ is a representative selection over set families : $\Psi\left(\left(A_{i}\right)_{i \in I}\right)=_{\text {def }}\left\{\cup_{i \in I}\left\{a_{i}\right\} / a_{i} \in A_{i}\right\}$

In the example of the vending machines, it holds that :

$$
\begin{aligned}
& \Psi\left(O U T_{M 2}(\operatorname{coin})\right)=\Psi(\{\{\text { T-button }\},\{\text { C-button }\}) \\
& =\{\{\mathrm{T} \text {-button, C-button }\}\}
\end{aligned}
$$

Intuitively, must $_{F}(\hat{\sigma})$ is composed of sets $\mathbf{A}$ verifying for all state $\mathrm{s}$ in $\mathrm{S}$ reachable by $\sigma$ there exists an action a in $\mathbf{A}$, such that $s \stackrel{a}{\Rightarrow}$.

Consequently, it can be easily shown that the domain $D^{\prime}$ attached to the resulting success tree verifies: $\quad D^{\prime} \sqsubseteq S[\operatorname{Trace}(S) \times \mathcal{P}(L)]$

Particularly, D' is a set of properties satisfied by $\mathrm{S}$, hence :

$$
\begin{aligned}
\text { Test }(\mathbf{F}, \mathbf{I}) \text { outputs "success" } & \left.\Leftrightarrow I \leq_{D^{\prime}} S \text { (theo. } 31\right) \Leftrightarrow I \leq_{\text {Trace }(S) \times \mathcal{P}(L)} S \text { (pro. 31-2) } \\
& \Leftrightarrow I \text { conf } S \text { (by def,) }
\end{aligned}
$$

Example : success trees F1, F2 and F3 from M1, M2 and M3 are depicted in Figure 2.

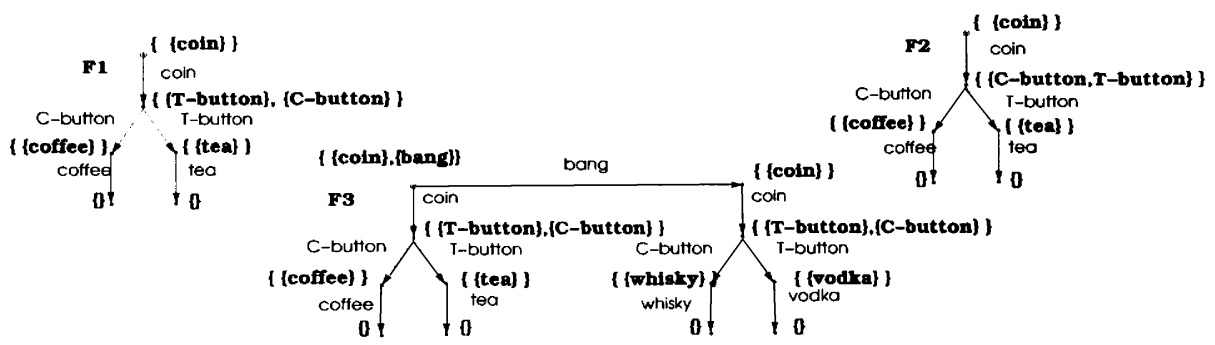

Figure 2 success trees

\section{TEST SYMMETRIES}

This section explains how to introduce the notion of symmetries in the field of testing. 


\subsection{Definition}

Informally, symmetries express assertions such as : M1 behaves in the same manner for coffee or tea, or else, after having performed bang, M3 delivers whisky instead of coffee and vodka instead of tea.

This action changing is expressed by a bijective renaming $\varphi$ over L. $\varphi$ is extended to action sequences and sets by :

$$
\varphi(\epsilon)=\epsilon \quad \varphi\left(a_{1} \ldots a_{n}\right)=\varphi\left(a_{1}\right) \ldots \varphi\left(a_{n}\right) \quad \varphi(\mathbf{A})=\{\varphi(a) / a \in \mathbf{A}\}
$$

Two states in the LTS are symmetrical iff they behave in the same way up to $\varphi$. As the behaviour is defined in terms of testable properties, a binary relation $T_{\varphi}$ over states is a test symmetry up to $\varphi$ iff :

$$
s T_{\varphi} s^{\prime} \Rightarrow \forall \sigma \in L^{*}, \forall \mathbf{A} \subseteq L: s \text { after } \sigma \text { must } \mathbf{A} \Leftrightarrow s^{\prime} \text { after } \varphi(\sigma) \text { must } \varphi(\mathbf{A})
$$

Example : In the example of the vending machine, M1, let $f$ be the renaming :

\begin{tabular}{l|l|}
\hline$a \mid$ coffee $\mid$ tea $\mid$ C-button $\mid$ T-button $\mid$ other action $x \mid$ \\
$|f(a)|$ tea $\mid$ coffee $\mid$ T-button |C-button
\end{tabular}

$T_{f}=\{(1,1),(2,2),(3,4),(5,6),(4,3),(6,5)\}$ is a test symmetry up to $f$ among states in M1.

If the LTS is deterministic, a test symmetry can be easily extended to sequences : two action sequences are related by $T_{\varphi}$ iff they reached two states related by $T_{\varphi}$. Formally : $\sigma T_{\varphi} \sigma^{\prime} \Rightarrow \forall \sigma_{1} \in L^{*}, \forall \mathbf{A} \subseteq L: B$ after $\sigma . \sigma_{1}$ must $\mathbf{A} \Leftrightarrow B$ after $\sigma^{\prime} . \varphi\left(\sigma_{1}\right)$ must $\varphi(\mathbf{A})$

Finally, the definition of test symmetries over any LTS is given by :

Definition $41 T_{\varphi} \subseteq L^{*} \times L^{*}$ is a test symmetry up to $\varphi$ upon $B$ iff :

$$
\begin{aligned}
\sigma T_{\varphi} \sigma^{\prime} \Rightarrow & \forall \mathbf{A} \subseteq L: B \text { after } \sigma \text { must } \mathbf{A} \Leftrightarrow B \text { after } \sigma^{\prime} \text { must } \varphi(\mathbf{A}) \\
& \text { and } \forall a \in L: \sigma . a T_{\varphi} \sigma^{\prime} \cdot \varphi(a)
\end{aligned}
$$

Example : $T_{f}=_{\text {def }}\{(\sigma, f(\sigma)) / \sigma \in \operatorname{Trace}(M 1)\} \cup\left\{\left(\sigma, \sigma^{\prime}\right) / \sigma, \sigma^{\prime} \notin \operatorname{Trace}(M 1)\right\}$ is a test symmetry up to $f$ over M1 and M2. Furthermore, if $g$ is defined by :

\begin{tabular}{l}
\hline$a \mid$ whisky $\mid$ coffee | tea | vodka | other action $x \mid$ \\
\hline$g(a) \mid$ coffee $\mid$ whisky | vodka | tea | \\
\hline
\end{tabular}

then $T_{g}={ }_{\text {def }}\{(\sigma$, bang.g $(\sigma))$ or $($ bang. $g(\sigma), \sigma) / \sigma$, bang.g $(\sigma) \in \operatorname{Trace}(M 3)\} \cup$ $\left\{\left(\sigma, \sigma^{\prime}\right) / \sigma, \sigma^{\prime} \notin \operatorname{Trace}(M 3)\right\}$ is a test symmetry up to g over M3.

\subsection{Group of symmetries and equivalence relation}

Let $T_{\varphi}$ be a test symmetry up to $\varphi$ over $\mathrm{B}, T_{\psi}$ be a test symmetry up to $\psi$ over $\mathrm{B}$. An homomorphism maps permutations to test symmetries:

- ID $=\left\{(\sigma, \sigma) / \sigma \in L^{*}\right\}$ is a test symmetry up to the identity renaming, over B.

- $T_{\varphi} \circ T_{\psi}$ is a test symmetry up to $\psi \circ \varphi$ over $\mathrm{B}$.

- $T_{\varphi}^{-1}$ is a test symmetry up to $\varphi^{-1}$ over $\mathrm{B}$. 
So, group structure can be brought to test symmetries. A group of test symmetries is a set of test symmetries containing ID and closed for the composition and inversion of relations. For each group $\mathcal{G}$ of test symmetries, a relation "is symmetrical to", denoted $\equiv$, can be defined as :

$$
\forall \sigma, \sigma^{\prime} \in L^{*}: \sigma \equiv \sigma^{\prime} \stackrel{\text { def }}{\Leftrightarrow} \exists T_{\varphi} \in \mathcal{G}, \sigma T_{\varphi} \sigma^{\prime}
$$

By using group laws, it holds that : $\equiv$ is an equivalence relation.

Example : $G_{1}=_{\text {def }}\left\{I D, T_{f}\right\}$ et $G_{3}=$ def $\left\{I D, T_{g}\right\}$ are groups of test symmetries, we note the attached equivalence relations respectively $\equiv_{1}$ and $\equiv_{3}$.

\section{A SYMMETRICAL SELECTIVE GENERATION}

First, let us assume the following hypothesis : I and S have common test symmetries, that is, there exists $\mathcal{G}$ such that :

$\mathcal{G}$ is a group of symmetries over I and over $S$

The induced relation "is symmetrical to" is denoted $\equiv$. The implementation relation to be tested is $\leq_{D}$ and $\mathrm{F}$ is a success tree attached to $\mathrm{D}$. The goal of this section is to exploit symmetries of $\mathcal{G}$ in order to find a domain D' verifying :

\begin{tabular}{l}
\hline $\mathrm{D}^{\prime} \subseteq \mathrm{D}(\mathrm{C} 1) \quad I \leq_{D} S \Leftrightarrow I \leq_{D^{\prime}} S(\mathrm{C} 2)$ \\
$\mathrm{D}^{\prime}$ can be represented by a success tree $\mathrm{F}^{\prime} \quad$ (C3)
\end{tabular}

Indeed, $(\mathrm{C} 1)$ implies $\mathrm{F}^{\prime}$ smaller than $\mathrm{F}$ implies the canonical tester from $\mathrm{F}^{\prime}$ is smaller than the canonical tester from $F$.

Furthermore, if Test $(\mathbf{F}, \mathbf{I})$ is valid, that is, D is a domain of properties verified by $\mathrm{S}$, then as a subset, $\mathrm{D}^{\prime}$ is also a domain of properties verified by $\mathrm{S}$, hence :

Test(F',I) outputs "success" $\Leftrightarrow I \leq_{D^{\prime}} S$ (theorem 31) $\Leftrightarrow I \leq_{D} E$ (condition (C2))

In part one, the candidate domains are defined as sets of representatives w.r.t. $\equiv$, i.e., domains whose intersections with each equivalence classes in $D / \equiv$ is a singleton. By definition, these domains satisfy (C1). Moreover, they are also shown to fulfill (C2).

Part two provides a construction of a quotient success tree from the success tree representing $\mathrm{D}$. The domain attached to the quotient success tree is a candidate domain. Hence, this domain fulfill conditions (C1) to (C3).

\subsection{Symmetrical implementation relations}

三 is extended to pairs $(\sigma, \mathbf{A})$ by $:(\sigma, \mathbf{A}) \equiv\left(\sigma^{\prime}, \mathbf{A}^{\prime}\right) \stackrel{\text { def }}{\Leftrightarrow} \exists T_{\varphi} \in \mathcal{G}: \sigma T_{\varphi} \sigma^{\prime}$ et $\mathbf{A}^{\prime}=\varphi(\mathbf{A})$

The symmetrical implementation relation consists in testing only a single property by classes of symmetrical properties. So, let us consider a domain D' of representatives in D w.r.t. $\equiv$ ( building a set of representative is selecting a member in each classes ). The symmetrical implementation relation, denoted $\left(\leq_{D} / \equiv\right)$, is defined by :

\section{Definition 51 (Symmetrical implementation relation) $I\left(\leq_{D} / \equiv\right) S \stackrel{\text { def }}{\Leftrightarrow} I \leq_{D^{\prime}} S$}

This definition does not depend on the chosen representative domain. Indeed, if $\mathrm{E}$ is another set of representatives, it holds that : $I \leq_{D^{\prime}} S \Leftrightarrow I \leq_{E} S$. 
By definition, if a testable property $\mathrm{P}$ is satisfied by $\mathrm{S}$ (resp. I), then all properties symmetrical to $\mathrm{P}$ are also satisfied by $\mathrm{S}$ (resp. I). Hence :

Theorem 51 If (H1) is verified then :I $\leq_{D} S \Leftrightarrow I\left(\leq_{D} / \equiv\right) S$

\subsection{Quotient success trees}

By definition, a success tree $F^{\prime}$ is a quotient success tree of $F$ w.r.t $\equiv$ iff the domain D' attached to F' is a representative domain w.r.t. $\equiv$ of the domain D.

This relationship is illustrated by the following diagram :

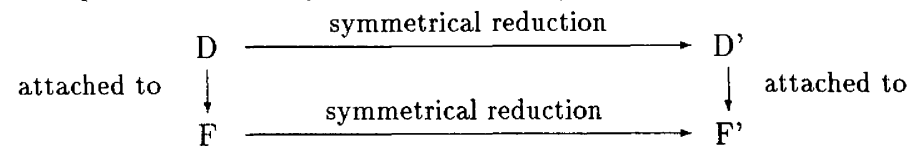

As some domains can not be represented by success trees, we have to show that such a quotient success tree exists. The proof is achieved by construction :

The quotient success tree, $\mathrm{F}^{\prime}$, is built level by level : level $\mathrm{k}$ is constructed from lowers levels. Formally, a level series $\left(F_{k}^{\prime}\right)_{k \geq 0}$ is defined inductively by :

Initialization $\mathbf{k}=\mathbf{0}$ : let us consider $D_{0}={ }_{\operatorname{def}}\left\{(\epsilon, \mathbf{A}) / \mathbf{A} \in\right.$ must $\left._{F}(\hat{\boldsymbol{\epsilon}})\right\}$, and $D_{0}^{\prime}$ a set of representatives in $D_{0}$ w.r.t. $\equiv . F_{0}^{\prime}$ is the success tree reduced to the root $\hat{\epsilon}$, and such that must $_{F_{0}^{\prime}}(\hat{\epsilon})=_{\text {def }}\left\{\mathbf{A} /(\epsilon, \mathbf{A}) \in D_{0}^{\prime}\right\}$.

Induction step : $F_{k+1}^{\prime}$ is built from $F_{k}^{\prime}$ according to the following operations :

1. Next $t_{k}$ is the domain of pairs $(\sigma . a, \mathbf{A})$ such that

- $\hat{\sigma}$ is a leaf of $F_{k}^{\prime}$,

- $\mathbf{A} \in \operatorname{must}_{F}(\widehat{\sigma . a})$ and $a \in \cup_{A \in m_{u s t} F_{k}^{\prime}} \mathbf{A}$,

- $\forall \sigma^{\prime} \in \operatorname{Trace}\left(F_{k}^{\prime}\right), \forall \mathbf{A}^{\prime} \in \operatorname{must}_{F_{k}^{\prime}}\left(\hat{\sigma}^{\prime}\right):(\sigma . a, \mathbf{A}) \not \equiv\left(\sigma^{\prime}, \mathbf{A}^{\prime}\right)$

2. $D_{k+1}^{\prime}$ is a set of representatives in Next $k$ w.r.t. $\equiv$,

3. $F_{k+1}^{\prime}$ is the success tree $F_{k}^{\prime}$ plus the level attached to $D_{k+1}^{\prime}$, that is : $\forall(\sigma . a, \mathbf{A}) \in$ $D_{k+1}^{\prime}$ : state $\widehat{\sigma . a}$ and transition $(\hat{\sigma}, a, \widehat{\sigma . a})$ are added in $F_{k+1}^{\prime}$, and $\mathbf{A}$ is added in must $_{F_{k+1}^{\prime}}(\widehat{\sigma . a})$

The limit of this series is the quotient success tree, $\mathrm{F}^{\prime}$. The demonstration is performed inductively by showing that for all $\mathrm{k}, F_{k}^{\prime}$ is a quotient success tree of $F_{k}$ (The subtree of $\mathrm{F}$ whose all node have a depth $\leq k$ ).

We note that this construction does not require that $\mathrm{F}$ is finite. Thus, quotient (and sometimes finite) success tree of infinite success trees can be considered. However, if $\mathrm{F}$ is finite, then the previous construction becomes an algorithm building automatically the quotient success tree from the initial success tree $F$.

Remark : the algorithm complexity relies strongly on determining the relation $\equiv$. Since the size of groups of symmetries may be important, computing $\equiv$ may be very hard. However, some particular kind of groups can be handled simply, as shown by some formalisms dedicated to symmetrical systems [Lam91]. 
Example : Quotient success trees F1' and F2' of F1 and F2 w.r.t. $\equiv_{1}$ and F3' of F3 w.r.t. $\equiv_{3}$ are depicted in Figure 3 .
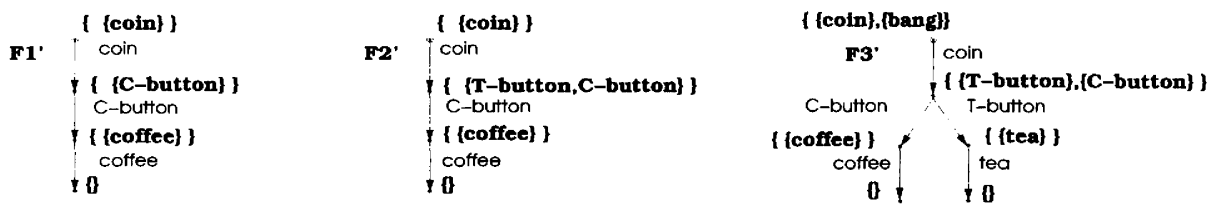

Figure 3 Quotient success trees

\section{THREE SORTS OF SELECTIVE GENERATION}

The operational application of the method requires to clarify two points :

- how to detect test symmetries over a partially-known implementation? The exploitation of structural information will allow us to determine test symmetries. There are three kind of informations : architectural, data and state symmetries.

- how to compute a quotient success tree when the initial one is infinite? We will explain how to compute a finite-branching quotient success tree from an infinitebranching success tree in case of data symmetries and how to compute finite-depth success tree from an infinite-depth success tree.

To study both problems, a formal description $\mathrm{P}$ in the Process Algebra CCS [Mil80] is assumed to be given. By the operational semantics of CCS, a L.T.S., B, can be derived. Both problems can be reformulated as :

the detection problem : how to detect test symmetries over $\mathrm{B}$, if $\mathrm{B}$ is not available, ( $P$ plays the role of the implementation).

The computation problem : how to compute the quotient success tree from $P$ when the initial success tree cannot be used ( $\mathrm{P}$ plays the role of the specification).

\subsection{Architectural symmetries}

\section{Detection problem}

An architecture of $\mathrm{n}$ parallel processes can be expressed by the following CCS term :

$$
\left(E_{1}\left[I_{1}\right]\left|E_{2}\left[I_{2}\right]\right| \ldots\left|E_{i}\left[I_{i}\right]\right| \ldots \mid E_{n}\left[I_{n}\right]\right) \backslash L_{\text {int }} \quad \text { where }
$$

$E_{i}$ is CCS expression describing the behaviour of the $i^{t h}$ isolated process, $I_{i}$ is a renaming function describing the interface of the $i^{\text {th }}$ process, and $L_{\text {int }}$ is the set of action only used for the internal synchronization among processes. $L_{\text {int }}$ expresses the encapsulation of actions.

In this context, an architectural symmetry is a pair $\left(\varphi_{I}, \varphi_{A}\right)$ such that $: \varphi_{I}$ is a permutation among process indexes, $\{1, \ldots, n\}, \varphi_{A}$ is a bijective renaming among actions, and $\left(\varphi_{I}, \varphi_{A}\right)$ leave the architecture unchanged :

$E_{i}$ and $E_{\varphi_{I}(i)}$ are syntactically equal and $I_{\varphi_{I}(i)}=\varphi_{A} \circ I_{i}$ and $\varphi_{A}\left(L_{i n t}\right)=L_{i n t}$ 
The set of architectural symmetries is a group, and each architectural symmetries can be turned into a test symmetry :

If $\left(\varphi_{I}, \varphi_{A}\right)$ is an architectural symmetries then $T_{\varphi_{A}}=\left\{\left(\sigma, \varphi_{A}(\sigma)\right) / \sigma \in L^{*}\right\}$ is a test symmetries up to $\varphi_{A}$.

\section{Computation problem}

The number of architectural symmetries is always finite, and so is the number of induced test symmetries. Consequently, if the initial success tree is infinite then the quotient success tree is also infinite. However, architectural symmetries allows us to decrease the size of a finite success tree by applying the construction of section 5.2.

\subsection{Data Symmetries}

In order to represent data, the value-passing version of CCS described in [Mil89] will be used in this section. [Mil89] defines a semantics of this calculus by a translation into CCS without data. Besides, the obtained expression may be infinite-branching. Data symmetries will be introduced in the value-passing CCS. Then, a symmetrical translation of the value-passing expression is defined. The interest of the symmetrical translation is twofold : on the one hand, the symmetrical translation preserves the testable properties up to test symmetries, on the other hand, the obtained expressions are generaliy finite-branching. Consequently, if $F^{\prime}$ is the success tree built from the result of the symmetrical translation, then $F^{\prime}$ is a quotient success tree and $F^{\prime}$ is likely to be finite-branching.

\section{Value-passing CCS}

Let Val be a set of values, and $\mathrm{X}$ a finite set of variables.

The value-passing is described by the following expressions : $a(x)$ is the input action which inputs a value from gate a and stores it in $\mathrm{x}$, and $\bar{a}(e)$ is the output action which outputs the result of expression e to gate a.

Data processing is expressed by the operator : if $\mathrm{b}$ then $\mathrm{P}$ with $\mathrm{b}$ boolean expression. If the result of $\mathrm{b}$ is true then the process behaves like $\mathrm{P}$ else it performs nothing.

The system expressions must not contain free variables, that is, each variable $x$ occuring free in a boolean or output expression is bounded by an input $a(x)$.

Example : Let $X=\{x, y\}$ and $V a l=\mathbf{R}$, the agent Max receiving two value from gate in and returning their maximum to gate out is expressed by :

$$
\text { Max } \stackrel{\text { def }}{=} \operatorname{in}(x) . i n(y) .(\text { if } x>y \text { then } \overline{\text { out }}(x) .0+\text { if } x \leq y \text { then } \overline{\text { out }}(y) .0)
$$

[Mil89] proposes a semantics of this calculus via a translation into basic CCS (CCS without data). It is based on the study of all the possible assignments of variables. An assignment is a partial function from variables to values. Let $f$ and $g$ be two assignments :

- $\operatorname{Dom}(f)$ is the definition domain of $f$,

- $f=g$ iff $\operatorname{Dom}(f)=\operatorname{Dom}(g)$ and for all $x$ in $\operatorname{Dom}(f): f(x)=g(x)$,

- $\emptyset$ is the assignment such that $\operatorname{Dom}(f)=\emptyset$,

- $f[v / x]$ is the assignment such that $\operatorname{Dom}(f[x / v])=\operatorname{Dom}(f) \cup\{x\}$ and $f$ is redefined at $x$ by $f(x)=v$ 
$f$ is said to be total iff $\operatorname{Dom}(f)=\mathrm{X}$. If $\mathrm{b}$ is an expression, $b . f$ denotes the expression where each variable $x$ is substituted by the value $f(x)$. Moreover, an evaluation function eval is assumed to be available. if $f$ is a total assignment, $\mathrm{b}$ a boolean expression and e an output expression then eval (b.f) is either true or false and eval (e.f) is a value in val. The input of a value $\mathrm{v}$ is denoted by $a_{v}$ and the output by $\overline{a_{v}}$ in basic CCS.

The translation, defined in this section, differs a little from the one proposed by [Mil89], since assignments are performed in a lazy way. However, the result is the same. $\operatorname{Trad}(f, P)$ the translation of $\mathrm{P}$, under assignment $f$, is defined by structural induction :

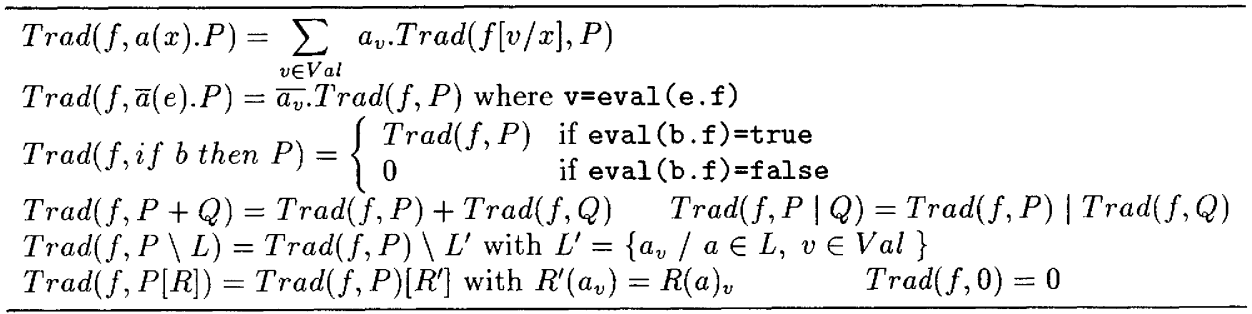

The translation over the expression describing the system is called by $\operatorname{Trad}(\emptyset, P)$.

To simplify, two restrictions are added over the considered value-passing CCS :

- the operator $\stackrel{\text { def }}{=}$ is forbidden, (no recursion is allowed).

- an input variable can not be used twice, for instance, expression $a(x) \ldots a(x) . P$ must be rewritten in $a(x) \ldots . a\left(x^{\prime}\right) \cdot P\left[x^{\prime} / x\right]$.

Example : $\operatorname{Trad}(\emptyset, M a x)=\sum_{v 1 \in \mathbb{R}} i n_{v 1} \cdot\left(\sum_{v 2<v 1} i n_{v 2} \cdot\left(\overline{\text { out }_{v 1}} \cdot 0+0\right)+\sum_{v 1 \leq v 2} i n_{v 2} \cdot\left(0+\overline{\text { out }_{v 2}} \cdot 0\right)\right)$

\section{Detection problem}

The system is represented by an expression $\mathrm{P}$ in value-passing CCS. $b_{1}, \ldots, b_{n}$ denote the boolean expressions and $e_{1}, \ldots, e_{m}$ the output expressions occuring in $\mathrm{P}$.

A data symmetry is a permutation $\varphi$ over Val such that :

1. $\forall f$ total assignment, $\forall 1 \leq i \leq n: \operatorname{eval}\left(b_{i} . f\right)=\operatorname{eval}\left(b_{i} \cdot(\varphi \circ f)\right)$

2. $\forall f$ total assignment, $\forall 1 \leq j \leq m: \varphi\left(\operatorname{eval}\left(e_{j} . f\right)\right)=\operatorname{eval}\left(e_{j} .(\varphi \circ f)\right)$

The whole set of data symmetries is a group and can be turned into test symmetries:

- $\varphi$ is extended to ground actions by :

$$
\varphi\left(a_{v}\right)=\operatorname{def}_{\operatorname{def}} a_{\varphi(v)} \text { and } \varphi\left(\overline{a_{v}}\right)=_{\operatorname{def}} \overline{a_{\varphi(v)}} \quad \text { and } \quad \varphi(\tau)=_{\operatorname{def}} \tau
$$

- $T_{\varphi}=_{\text {def }}\left\{(\sigma, \varphi(\sigma)) / \sigma \in L^{*}\right\}$ is a test symmetry up to $\varphi$ over $\operatorname{Tr} a d(\emptyset, P)$.

Example : Data symmetries in Max are strictly increasing bijections over $\mathbb{R}$. 


\section{Computation problem}

The guideline is to translate only one case per symmetrical cases. The notion of symmetrical cases is specified by a symmetry relation, denoted $\equiv$, among assignments :

$\forall f, g$ assignments : $f \equiv g \stackrel{\text { def }}{\Leftrightarrow} \exists \varphi$ data symmetry : $\varphi \circ f=f^{\prime}$

$\equiv$ is an equivalence relation and a set of assignment representatives can be built by the sequence $\left(A T_{i}\right)_{0 \leq i \leq|X|}: A T_{0}=_{\text {def }}\{\emptyset\}$ and for all $\mathrm{i}$ in $\{0, \ldots,|X|-1\}$

$A T_{i+1}$ is a set of representatives w.r.t $\equiv$ for $\left\{f[v / x] / f \in A T_{i}, x \notin \operatorname{Dom}(f)\right.$ and $v \in$ Val $\}$.

$A T_{|X|}$ is a set of assignment representatives w.r.t. $\equiv$, denoted $A T_{\text {sym }}$.

The symmetrical translation, denoted $\operatorname{Trad}_{\text {sym }}$, is a translation where only the assignments in $A T_{\text {sym }}$ are authorized. Its definition is the same that Trad except for the rule:

$\operatorname{Trad}_{s y m}(f, a(x) \cdot P)=\sum_{v: f[v / x] \in A T_{s y m}} a_{v} \cdot \operatorname{Trad}_{s y m}(f[v / x], P)$

The interest of the symmetrical translation lies in the property illustrated by the following diagram :

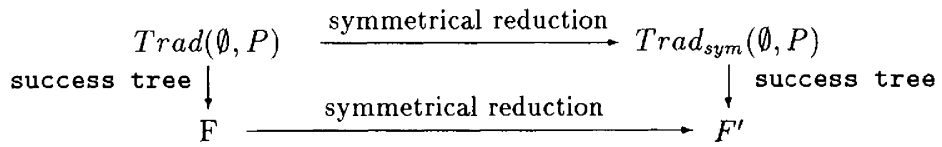

Indeed, it can be shown that the symmetrical translation preserves all the testable properties modulo data symmetries :

1. $\operatorname{Trad}(\emptyset, P)$ after $\sigma$ must $\mathbf{A} \Rightarrow \exists \varphi$ symmetry: $\operatorname{Trad}_{\text {sym }}(\emptyset, P)$ after $\varphi(\sigma)$ must $\varphi(\mathbf{A})$

2. $\operatorname{Trad}_{\text {sym }}(\emptyset, P)$ after $\sigma$ must $\mathbf{A} \Rightarrow \operatorname{Trad}(\emptyset, P)$ after $\sigma$ must $\mathbf{A}$

Consequently, the success tree built from $\operatorname{Trad}_{s y m}(\emptyset, P)$ is a quotient success tree of the success tree attached to $\operatorname{Trad}(\emptyset, P)$.

Example : In the case of Max, $A T_{\text {sym }}={ }_{\text {def }}\left\{f_{1}, f_{2}, f_{3}, f_{4}, f_{5}\right\}$ where $f_{1}: x \rightarrow 0, f_{2}: y \rightarrow 0$

$f_{3}: x \rightarrow 0, y \rightarrow 1, f_{4}: x \rightarrow 1, y \rightarrow 0$ and $f_{5}: x \rightarrow 0, y \rightarrow 0$, hence :

$\operatorname{Trad}_{\text {sym }}(\emptyset, M a x)=i n_{1} \cdot i n_{0} \cdot\left(\right.$ out $\left._{1} \cdot 0+0\right)+i n_{0} \cdot\left(i n_{1} \cdot\left(0+o u t_{1} \cdot 0\right)+i n_{0} \cdot\left(0+o u t_{0} \cdot 0\right)\right)$

\subsection{State Symmetries}

\section{Detection problem}

State symmetries express assertions such as : after sequence $\sigma$ the system is in the same state that after $\sigma^{\prime}$. Formally, it corresponds to the test symmetries up to the identity defined by : $T_{i d}=\left\{\left(\sigma . \sigma_{e}, \sigma^{\prime} . \sigma_{e}\right) / \sigma_{e} \in L^{*}\right\}$.

State symmetries are more difficult to detect than architectural or data symmetries. However, they can be stated, if some states in the system are observable.

\section{Computation problem}

State symmetries are useful to cut loops which make success tree $F$ depth infinite.

Since the depth of $F$ is infinite, it is impossible to first build $F$ and then build the quotient success tree F'. On the other hand, the construction of section 5.2, needs only 
the $k^{\text {th }}$ level of $\mathrm{F}$ to build the $k^{\text {th }}$ level of $\mathrm{F}^{\prime}$. Hence, the construction of $\mathrm{F}$ can be overlaped incrementally with the construction of $F^{\prime}$ : At each step of the following procedure, level $\mathrm{k}$ of $\mathrm{F}$ is built from the CCS expression describing the system and then level $\mathrm{k}$ of the quotient success tree is derived.

If there exists a finite-depth quotient success tree then this procedure will terminate although $\mathrm{F}$ is infinite.

Example : Suppose that M1 is reinitialisable, we note Mr the new machine :

$$
M r \stackrel{d e f}{=} \text { coin.(C-button.coffee. } M r+\text { T-button.tea. } M r)
$$

That is, after coin.T-button.tea

or coin.C-button. coffee, the system is in the initial state. So, we have the following state symmetries $T_{I d}^{c}$ and $T_{I d}^{t}$ such that :

$\forall \sigma \in L^{*}: \quad \epsilon . \sigma T_{I d}^{c}$ coin.C-button.coffee. $\sigma$

$\epsilon . \sigma T_{I d}^{t}$ coin.T-button.tea. $\sigma$

Figure 4 depicts the construction steps of the quotient success tree Mr'.

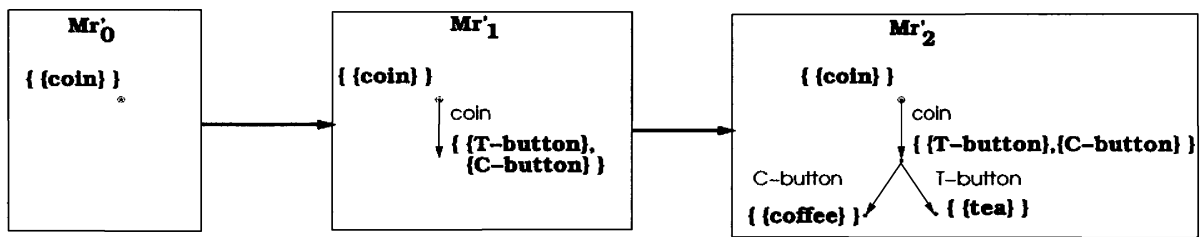

Figure 4 Construction steps

\section{CONCLUSION}

The paper have proposed a mean to drastically reduce the complete test suite required for a implementation relation : the symmetrically selective test generation. Within the selected theoretical framework developed by [Bri88, Tre92], test symmetries have been introduced. Their exploitation results in a reduction of the size of the success tree and so the canonical tester representing the complete test suite.

For the proposed method, system symmetries are needed as extra informations, and the test selection is performed over the success tree, at the beginning of the generation.

The latter point is very interesting since sometimes it allows us to avoid the generation of infinite test suites. The first point has an advantage : as extra and not external informations are used, the implementation relation is not weakened by the reduction. On the other hand, it has a drawback, the extra informations must be detected. Consequently, we have explained how to detect and exploit symmetries in three cases : architectural, data and state symmetries. Data symmetries are demonstrated to overcome problems caused by infinite branching in some cases and state symmetries appear to be a good solution to cut infinite-length test cases.

In this paper, symmetry processing is not detailed : we suppose that the designer is able to provide symmetries and to implement a decision procedure for the relation "is symmetrical to". However, some tools already manage symmetry processing [ID93, 
GCH91]. Consequently, their integration into the symmetrically selective test generation is being considered. Furthermore, these tools deal with architectural symmetries [GCG94], and their scope extension to data and state symmetries would be very interesting.

\section{REFERENCES}

[Bri88] E. Brinksma. A theory for the derivation of tests. In S. Aggrawal and K.Sabani, editors, Protocol Specification Testing and Verification, volume VIII. Elsevier Science Publishers B.V., North-Holland, 1988.

[BTV91] E. Brinksma, J. Tretmans, and L. Verhaard. A framework for test selection. In Proc. of the 11th Symposium on Protocol Specification, Testing Specification, Testing and Verification, Stockholm, June 17-20 1991. international IFIP WG6.1.

[ECJ93] T. Filkorn E.M. Clarke and S. Jha. Exploiting symmetry in temporal logic model checking. In Computer Aided Verification, pages 451-462. Lecture Notes in Computer Science 697, June-July 1993.

[EE93] A. Sistla E. Emerson. Symmetry and model checking. In Computer Aided Verification, pages 463-478. Lecture Notes in Computer Science 697, June-July 1993.

[EE95] A. Sistla E. Emerson. Utilizing symmetry when model checking under fairness assumptions: an automata-thoeirc approach. In Computer Aided Verification, pages 279-292. Lecture Notes in Computer Science 939, June-July 1995.

[GCG94] G. Franceschinis G. Chiola and R. Gaeta. Modeling symmetric computer architectures by swns. In Application and Theory of Petri Nets, pages 139-158. Lecture Notes in Computer Science, 1994.

[GCH91] G. Franceschinis G. Chiola, C. Dutheillet and S. Haddad. On well-formed coloured nets and their symbolic reachability graph. In High-Level Petri Nets, pages 373-396. Springer-Verlag, 1991.

[Hen85] M. Hennessy. Acceptance trees. Journal of the Association for Computing Machinery, 32(4):896-928, October 1985.

[ID93] C. Ip and D. Dill. Better verification through symmetry. In Int. Symp. on Computer Hardware Description language and their Application, April 1993.

[Lam91] Claude Dutheillet Lamonthezie. Symétrie dans les Réseau Colorés. PhD thesis, Université Paris 6, 1991.

[Mil80] Robin Milner. A Calculus of communicating Systems, chapter Syntax and Semantic of CCS, pages 77-78. LNCS 92. Springer-Verlag, 1980.

[Mil89] Robin Milner. Communication and Concurrency. Prentice Hall, 1989.

[PHJ91] L.O. Jepsen P. Huber, A.M. Jensen and K. Jensen. Reachability trees for highlevel petri nets. In High-Level Petri Nets, pages 319-350. Springer-Verlag, 1991.

[Sch94] K. Schmidt. Symmetries of petri nets. Informatik-Berichte 33, HumboltUniversität zu Berlin, 1994.

[Tre89] J. Tretmans. Test case derivation from lotos specification. In Proc. 2nd International Conference on Formal Description Techniques. FORTE'89, Vancouver, CANADA, 1989.

[Tre92] J. Tretmans. A Formal Approach to Conformance Testing. Phd thesis, University of Twente, The Netherlands, 1992. 


\section{APPENDIX 1 TEST GENERATION}

This appendix details the derivation and the application of a complete test suite, the canonical tester, w.r.t. $\leq_{D} . \mathrm{F}$ is assumed to be a success tree representing a domain $\mathrm{D}$. The derivation consists of three steps :

The canonical tester: The canonical tester $\mathrm{T}$ attached to $\mathrm{F}$ is the L.T.S. verifying :

- States in $\mathrm{T}$ are states $\hat{\sigma}$ of $\mathrm{F}$, the indexed states $\hat{\sigma}_{A}$ where $\mathbf{A} \in \operatorname{must}_{F}(\hat{\sigma})$, and dead, a deadlock state dead. $\hat{\epsilon}$ is the initial state of $\mathrm{T}$,

- The transition relation is ruled by ( $\hat{\sigma}$ is a state in $\mathrm{F}$ ) :

$$
\begin{aligned}
& \frac{\mathbf{A} \in \text { must }_{F}(\hat{\sigma})}{\left(\hat{\sigma}, \tau, \hat{\sigma}_{A}\right) \text { transition in T }}(\text { Choice }) \\
& \frac{a \in \mathbf{A} \text { and } \widehat{\sigma . a} \text { state in } \stackrel{F}{\left(\hat{\sigma}_{A}, a, \widehat{\sigma . a}\right) \text { transition in } \mathrm{T}}(\text { Action } 1) \quad \frac{a \in \mathbf{A} \text { and } \widehat{\sigma . a} \text { not a state of } F}{\left(\hat{\sigma}_{A}, a, \text { dea }\right) \text { transition in } \mathrm{T}}(\text { Action } 2)}{}
\end{aligned}
$$

The (Choice) rule represents the internal choice of the tester among properties to be tested. Rules (Actioni) represent the expecting observation of action a.

The property $\operatorname{out}_{F}(s) \subseteq U_{\mathbf{A} \in \text { foncF(s) }} \mathbf{A}$ of the success tree ensures that $\mathrm{T}$ is really a L.T.S., that is, all the states $\hat{\sigma}$ or $\hat{\sigma}_{A}$ are reachable. This means that no property in D is lost by $\mathrm{T}$.

Example : Figure 5 depict the canonical tester T3' attached to the quotient success tree F3'.
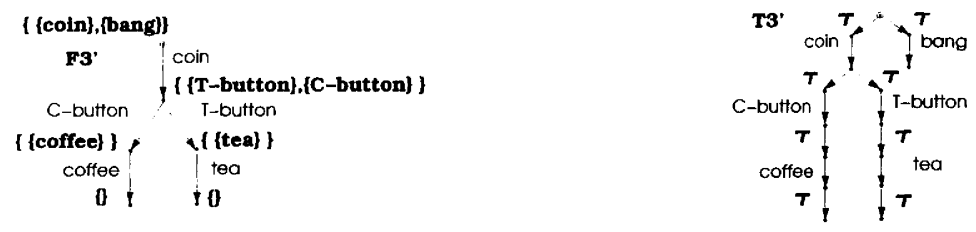

Figure 5 Canonical tester

Synchronous application to the implementation : Tests are run over the implementation by synchronization on common actions. The result of this synchronization is the L.T.S. T $\| I$ such that :

- states in $T \| I$ have the form $s \| s^{\prime}$ where $\mathrm{s}$ is a state in T and s' is a state in I. The initial state in $T \| I$ is $s_{0} \| s_{0}^{\prime}$ where $s_{0}$ is the initial state in T (i.e. $\hat{\epsilon}$ ) and $s_{0}^{\prime}$ the initial state in I.

- The transition relation is ruled by :

$$
\frac{s \stackrel{a}{\Rightarrow} p \quad \text { and } \quad s^{\prime} \stackrel{a}{\Longrightarrow} p^{\prime}}{s\left\|s^{\prime} \stackrel{a}{\Longrightarrow} p\right\| p^{\prime}}
$$

Verdict : the verdict is obtained by observing deadlocks. A deadlock is observed over a S.T.E. $B$ after a sequence $\sigma$ iff :

In this case, we note $B \stackrel{\sigma}{\Longrightarrow}$ deadlock.

$$
\exists s:(B \stackrel{\sigma}{\Longrightarrow} s \text { and } \forall a: s \stackrel{a}{\Rightarrow})
$$

The verdict is "success" iff all the deadlocks are caused by the tester :

$$
\forall \sigma \in L^{*}: T \| I \stackrel{\sigma}{\Rightarrow} \text { deadlock } \Rightarrow T \stackrel{\sigma}{\Rightarrow} \text { deadlock }
$$

